\title{
Diabetes and depression
}

\author{
Eon-Ju Jeon \\ Department of Internal Medicine, Catholic University of Daegu, School of Medicine, Daegu, Korea
}

\begin{abstract}
Diabetes and depression are serious chronic conditions. As a result of their increasing prevalence, diabetes and depression, together with population growth and aging, are public health issues. The rate of depression in adults with either type 1 diabetes or type 2 diabetes is high relative to the general population. The coexistence of diabetes and depression has attracted much interest. Although it is unclear whether diabetes and depression are causally linked, most studies have shown that the association between diabetes and depression might be bidirectional. Currently, emotional well-being is becoming an increasingly important aspect of diabetes care and self-management. Psychiatric disorders and diabetic distress among people with diabetes may increase the risk of diabetes complication and mortality. Thus, assessment and appropriate management of depression in people with diabetes should be considered to achieve psychological well-being and optimize medical outcomes.
\end{abstract}

Keywords: Depression; Diabetes; Mental Health

\section{INTRODUCTION}

Diabetes is a chronic disease with an increasing prevalence rate. According to reports from the International Diabetes Federation (IDF) in 2017, the number of people with diabetes aged 20-79 years was 424.9 million and was predicted to increase to 629 million [1]. Diabetes prevalence, deaths owing to diabetes, and healthcare expenditure owing to diabetes present a large social, financial, and health system burden worldwide. According to the World Health Organization (WHO), the number of people with depression globally in 2015 was 322 million (4.4\%) [2]. Depression is more common among females (5.1\%) than among males (3.6\%), and it has been increasing worldwide and currently is the leading cause of global

Received: April 22, 2018, Revised: June 7, 2018 Accepted: June 8, 2018

Corresponding Author: Eon-Ju Jeon, Department of Internal Medicine, Catholic University of Daegu School of Medicine,

33, Duryugongwon-ro 17-gil, Nam-gu, Daegu 42472, Korea

Tel: +82-53-650-4098, Fax: +82-53-651-4009

E-mail: ejjeon@cu.ac.kr mental and physical disability. In Korea, the prevalence of diabetes among adults aged $\geq 30$ years is $13.7 \%$ (4.8 million) according to the 2013-2014 Korean National Health and Nutrition Examination Survey [3]. In the 2016 Epidemiological Survey of Mental Disorders in Korea, the lifetime prevalence was $5.3 \%$ for mood disorders (7.2\% in females, 3.3\% in males) and 5.0\% for major depressive disorders (MDDs; $6.9 \%$ in females, $3.0 \%$ in males).

The importance of diabetes and mental health has recently been emphasized on. An individual with diabetes should be considered as a person who lives with diabetes rather than a patient with a disease. Mental health of diabetes was ignored because blood glucose control was more urgent during the last few decades. Diabetes, a chronic disease, can affect mental health and lead to psychosomatic disorders. A large number of people with diabetes experience diabetes distress, which refers to the negative emotions and burden of self-care related to life with diabetes. Depression is the most common psychiatric disorder associated with diabetes [4]. Depression is associated with non-adherence to diabetes self-management, including following dietary restrictions, medication com-

Copyright (C) 2018 Yeungnam University College of Medicine

This is an Open Access article distributed under the terms of the Creative Commons Attribution Non-Commercial License (http://creativecommons.org/licenses/by-nc/4.0/) which permits unrestricted non-commercial use, distribution, and reproduction in any medium, provided the original work is properly cited. 
pliance, and blood glucose monitoring, resulting in worse overall clinical outcomes [5]. Although it is possible that diabetes is associated with depression, depression remains underdiagnosed and undertreated in people with diabetes. Practice guidelines from IDF indicate that as individuals with diabetes are more likely affected by depression, there is a strong requirement for periodic assessment and monitoring of depression and other mental health conditions for more effective diabetes management [6]. In practice guidelines for the treatment of patients with MDD, American Psychiatric Association recommended in 2010 to monitor diabetic control, because fluctuations in fasting blood glucose may occur, when initiating antidepressant therapy or adjusting for dosage [7]. In Canada, screening for diabetes should be considered more frequently or earlier in people with additional risk factors for type 2 diabetes, such as psychiatric disorders including depression, bipolar disorders, and schizophrenia [8]. Lately, the American Diabetes Association (ADA) released a position statement to provide diabetes care providers with guidelines for psychosocial assessment and care of patients with diabetes [9]. The position statement emphasizes that psychosocial care should be provided to all individuals with diabetes, with the goal to optimize health outcomes and health-related quality of life. It provided routine monitoring and screening for diabetes distress, depression, anxiety, eating issues, and appropriate levels of social and family support. The purpose of this review is to summarize the current understanding of diabetes and depression, focusing on screening tools for depression in people with diabetes.

\section{Association between diabetes and depression}

The literature investigating the relationship of diabetes to psychiatric disorders continues to grow. These studies focus on the relationship between diabetes and depression, eating disorders, and anxiety disorders. The causal links between psychiatric disorders and diabetes are not well understood, however growing evidence suggests that there is a connection. Metabolic problems of diabetes may play a role in the development of depression. Persistent hyperglycemia and episodic severe hypoglycemia in diabetes can change the function, neurochemistry, or structure of the brain [10]. These conditions with non-diabetic factors and family coping might lead to depressive disorders, anxiety disorders, or eating disorders. There is increasing evidence that diabetes leads to changes in the white matter of the brain and that these abnormalities, if present in the frontal lobe, may play a role in the development of depression [10,11]. Biological changes related to depression include abnormal mechanisms of neurotransmitters such as norepinephrine, serotonin, and dopamine, hypothalamus-pituitary-adrenal (HPA) axis dysfunction, immunological changes in cytokines, vascular disease, decreases in neurotrophic substances, and atrophy of the frontal cortex or hippocampus[11,12]. A recent study investigated the single nucleotide polymorphisms of the genes for serotonin transporter, tryptophan hydroxylase 2, brain-derived neurotrophic factor, and the P2RX7 purinergic receptor [13]. The results supported the role of the P2RX7 rs2230912 G-allele in the development of depression and emphasized the importance of good glycemic control, acting as a potential protective factor for patients with diabetes. In addition, a large range of differences exists among individuals' vulnerabilities to the development of disorders under the same stress. The differential susceptibility may have been caused by genetic factors. The median age of depression onset is between 20s and 40s. More women than men are diagnosed with depression. The average age of depression onset suggests that the differences exist in the relationship between age and type 1 and type 2 diabetes. Type 1 and type 2 diabetes should be approached separately to identify their association with depression. Type 1 diabetes appears in childhood and early adulthood requiring daily insulin injections for life, while type 2 diabetes appears later in life, in mid-adulthood, requiring diet and lifestyle modifications, oral medication or insulin injections [14].

Depression is a condition characterized by episodes of depressed mood [15]. Each episode is characterized by mood lowering, reduction of energy, and decrease in activity. Depression should be distinguished from states of subjective distress and emotional disturbance, possibly interfering with social functioning and performance, arising during the period of adaptation to significant life changes or stressful life events such as death of a loved one. People living in modern environment are more likely to experience depressive symptoms compared with those living in the past environment. Mild depressive symptoms, such as sadness, empty mood, or irritability, are sometimes referred to as depression. Therefore, clinical depression can identify more people as having a de- 
pressive disorder. Depression in psychiatry is referred to as MDD [16].

An association between diabetes and depression was recognized as early as the 17th century, when the British physician Thomas Willis noted that diabetes frequently appeared in individuals who had experienced previous life stresses or sadness [17]. In a systematic review, the risk of progression to depression increased in the presence of diabetes and the risk of progression to diabetes increased in the presence of depression [18]. Diabetes and depression are thought to be complex and bidirectional, although it remains unclear. Most studies examining the relationship between diabetes and psychiatric disorders are cross-sectional in design. Whether the phenotype of depression in diabetes differs between people with or without diabetes is not known [14].

\section{Type 1 Diabetes and depression}

Type 1 diabetes is usually diagnosed in childhood and adolescence at a time of rapid psychological and physiological changes and potentially causes substantial disruption of normal development. Type 1 diabetes require a complex treatment regimen and frequent blood glucose measurements due to multiple insulin injections in prescriptions. These parameters impose dietary restrictions or activity limitations, resulting in a psychological burden. These may be different from the neurological disorder observed in type 2 diabetes. Studies examining the association between type 2 diabetes and depression have been relatively consistent; however, studies on type 1 diabetes have shown various results depending on the study $[19,20]$. Type 1 diabetes is associated with adjustment disorders, anxiety disorders, and eating disorders rather than depression. Families of diabetic patients showed worse family relationships among members, and the risk of subsequent psychiatric problems was higher [21]. There is also a possibility of developmental problems due to biological changes, such as inflammation and brain atrophy, and diagnosis at a young age [22,23]. Insulin-induced hypoglycemia may be associated with neurocognitive deficits observed in patients with earlyonset type 1 diabetes and may be a risk factor for MDD development. Hyperactivity of the HPA axis has been noted in both type 1 diabetes and MDD and provides a putative link between type 1 diabetes and MDD comorbidity [23]. Magnetic resonance imaging (MRI) of the brain of people with type 1 diabetes has shown that prefrontal glutamate-glutamine-gamma-aminobutyric acid levels are higher than in healthy control individuals, and these levels correlate with mild depressive symptoms [24]. Thickness of prefrontal cortical lesions was reduced in people with type 1 diabetes [25]. Basal ganglia and insula mediation of interoceptive awareness during hypoglycemia were altered in type 1 diabetes patients [26].

\section{Type 2 diabetes and depression}

The co-occurrence of type 2 diabetes and depression has attracted much research interest. If this association was causal, it would have profound implications for the prevention and treatment of these disorders. However, the association between diabetes and depression seems to be complex, and does not follow a simple cause and effect pattern. Type 2 diabetes precedes the onset of depression and the risk of developing depression within 1 year after diagnosis of diabetes is the highest [27-29]. Depressive disorders may increase the risk of type 2 diabetes. Thus, some aspects of the physiological changes associated with depression are consistent with diabetes causation. There are possible pathophysiological, biological, and behavioral links. First, depression as a cause of type 2 diabetes is associated with increased activity of the HPA axis and the sympathetic nervous system, resulting in increased release of cortisol and other glucocorticoids, catecholamines such as adrenaline and noradrenaline, growth hormone, and glucagon $[4,30,31]$. These lead to increase in glucose production and lipolysis, and decreased insulin sensitivity. Depression begins at a young age and is associated with decreased self-esteem, decreased physical activity, and increased risk of cardiovascular disease due to smoking and high calorie diet. At more advanced stages of type 2 diabetes, complications might cause an increased risk of depression or depressive symptoms. Antidepressant drugs might also have diabetogenic side-effects. Second, considering type 2 diabetes as a cause of depression, preclinical changes such as increased blood glucose or altered insulin concentrations might directly affect depression risk through biochemical mechanisms [4]. Diagnosis of diabetes is generally recognized as a negative event. Stress due to the chronicity of the process and complications related to the long duration of disease cause psychological and economic burdens. Third, diabetes and depression 
share common metabolic abnormalities. The so-called common-soil hypothesis posits that the non-causal association between them results from factors affecting both disorders including chronic inflammation, obesity, physical inactivity, and vascular risk. Cytokines such as interleukin (IL) 1 or 6 and tumor necrosis factor (TNF) are associated with inflammation $[4,14,32,33]$. Disruption of normal circadian rhythm is implicated in both depression and type 2 diabetes [34,35]. Lately, genome-wide association studies (GWAS) revealed the significant genetic overlap between depression and type 2 diabetes in twin registries [36].

\section{Screening for depression in people with diabetes}

Depressive symptoms and depressive disorders affect one in four patients with type 1 and type 2 diabetes [37]. The rate of treatment for those diagnosed and treated is approximately $15 \%$. Clinicians mainly refer patients to psychiatrists, when patients with diabetes are strongly suspicious of depression. In some cases, self-reported questionnaires have been conducted, when patients do not have prominent symptoms for depression. Several screening tests, including Beck Depression Inventory (BDI), Depressive Cognition Scale, Zung's Screening for Depression (SDS), Center for Epidemiologic Studies Depression (CES-D) Questionnaire, are used [38-41].

In a position statement from $\mathrm{ADA}$, all care providers should include queries about well-being in routine care. The situations in Table 1 should be referred to a mental health professional for assessment [9]. ADA recommends screening with standardized, well-validated tools for monitoring and evaluation of cognitive and diabetic complaints, depression, anxiety disorders, and eating disorders at the initial visit of people with diabetes (Table 2) [9]. Routine screening for depressive symptoms is indicated in high-risk populations, including people with prediabetes, particularly those who are overweight, type 1 and type 2 diabetes, and postpartum diabetes [9,42]. To detect problems early and prevent health deterioration, all people with diabetes should be evaluated on a periodic basis, going forward basis even if there is no patient-specific indication [43]. Physicians should consider annual screening for patients with diabetes, especially for those with a self-reported history of depression. In addition, an evaluation is recommended during major disease and life transitions, inclu-

Table 1. Situations that warrant referral of a person with diabetes to a mental health provider for evaluation and treatment [9]

If self-care remains impaired in a person with diabetes distress after tailored diabetes education

If a person has a positive screen on a validated screening tool for depressive symptoms

In the presence of symptoms or suspicions of disordered eating behavior, an eating disorder, or disrupted patterns of eating

If intentional omission of insulin or oral medication to cause weight loss is identified

If a person has a positive screen for anxiety or fear of hypoglycemia

If a serious mental illness is suspected

In youth and families with behavioral self-care difficulties, repeated hospitalizations for diabetic ketoacidosis, or significant distress

If a person screens positive for cognitive impairment

Declining or impaired ability to perform diabetes self-care behaviors

Before undergoing bariatric surgery and after if assessment reveals an ongoing need for adjustment support

Table 2. Measures for the evaluation of depression in the clinical setting [9]

\begin{tabular}{lll}
\hline Measure title & \multicolumn{1}{c}{$\begin{array}{c}\text { Validated } \\
\text { population }\end{array}$} & \multicolumn{1}{c}{ Description } \\
\hline PHQ-9 [46] & Adults & $\begin{array}{l}\text { 9-item measure of depressive symptoms (corresponding to criteria for major } \\
\text { depressive disorder) }\end{array}$ \\
BDI-II [38] & Adults & $\begin{array}{l}\text { 21-item questionnaire evaluating somatic and cognitive symptoms of depression } \\
\text { GDS [52] }\end{array}$ \\
CDI (current edition is CDI-2) [66] & Ydults (55-58 yr) & 15-item measure was developed to assess depression in older adults \\
\hline
\end{tabular}

PHQ-9, Patient Health Questionnaire; BDI-II, Beck Depression Inventory-II; GDS, Geriatric Depression Scale; CDI, Child Depression inventory. 
ding diagnosis of complications and significant changes in treatment (e.g., initiation of insulin pump or other forms of intensification) or in life circumstances (e.g., living arrangement, job, and significant social relationships), with prospective monitoring for 6 months [9,44]. In fact, psycosocial assessment is an important aspect of diabetes care, although it has been neglected due to the importance and urgency to control glucose levels. For example, diabetes care providers can ask whether there have been changes in the mood during the past 2 weeks or since their last visit and should consider asking whether there are new or different barriers to treatment and self-management, such as feeling overwhelmed or stressed by diabetes or other stress factors [9]. Professional diagnostic tests for mental health will be necessary for individuals with a positive evaluation screening. People with diabetes, as well as their families and caregivers should be evaluated. For adults $\geq 65$ years old, annual screening for cognitive impairment or dementia is indicated. It is hard to say which tool is the best. There are advantages and disadvantages depending on the situation of the patients and the purpose of evaluation. In Korea, although the reliability and validity of Korean Version of BDI-II have been studied for standardization of the tool for use in depression, it is necessary to develop a screening test that suits the characteristics of the Korean population [45].

\section{Patient Health Questionnaire-9 (PHQ-9)}

PHQ-9 is a tool widely used for depression screening in primary care and clinical research. It is a self-reporting test designed and assess the severity of depression, simply and quickly. PHQ-9 questions are based on the diagnostic criteria for depression from the Diagnostic and Statistical Manual of Mental Disorders (DSM)-IV and ask about patient's experience in the last 2 weeks. Questions interrogate the level of interest in doing things, whether individuals feeling down or depressed, difficulty with sleeping, energy levels, eating habits, self-perception, ability to concentrate, speed of functioning and thoughts of suicide with a score range from 0 (not at all) to 3 (nearly every day) [46,47]. It takes 3 minutes to complete. PHQ-2 is used to reduce time to respond to the questionnaire and to facilitate evaluation [48]. However, PHQ-2 has a higher rate of false-positive responses than other screening instruments for depression.

\section{Beck Depression Inventory-II (BDI-II)}

The original BDI, first published in 1961, consisted of 21 questions regarding how individuals have been feeling during the last week [49]. BDI-II, which was a 1996 revision of BDI, was developed according to DSM-IV, which changed many of the diagnostic criteria for MDD [38]. BDI-II items are rated on a four-point scale ranging from 0 to 3 based on the severity of each item. The maximum total score is 63 . It takes 10 minutes to complete. In several studies, this scale has been shown to be reliable and provides valid measurements for various groups [50]. Self-reported scales depend on the subjective symptoms of the patients. Therefore, scores may be exaggerated or minimized depending on the person completing the questionnaire.

\section{Geriatric Depression Scale (GDS)}

GDS, which was first developed in 1982, is a 30 -item selfreported assessment used to identify depression in the elderly [51]. A short version of GDS with 15 questions has been developed [52]. Diabetes and depression is an important health condition for the aging population. When depression occurs in the elderly, it may mimic dementia due to delayed psychomotor speed, decreased cognitive function, and subjective memory loss. Physical symptoms are also present in elderly people who are not depressed, therefore, it is difficult to distinguish between individuals with or without depression, and long time may be required to distinguish between these individuals. The question form requiring discrimination and selection should be avoided. Evaluation is desirable to reduce the number of factors for somatic symptoms, and to select "yes" or "no" for factors related to cognitive decline. In Korea, the Korean version of GDS is used. Although cutoff points were higher in Korea than in Western countries, GDS remains reliable and valid as a self-rate depression screening scale for elderly psychiatric patients [53]. In elderly people with impaired cognitive function, GDS is not feasible because validity is not maintained [54]. 


\section{Diagnosis for depression in people with diabetes}

Clinicians should consider annual screening of all people with diabetes, especially those with a self-reported history of depression, for depressive symptoms with age-appropriate depression screening, recognizing that further evaluation will be necessary for individuals with a positive screen [42]. The most widely used tools for diagnostic conformation of depression are the DSM-V by the American Psychiatric Association and International Classification of Disease 10th Revision (ICD-10) published by WHO [15,16,55,56]. Depression has a variety of subtypes within the upper category. Evaluation and treatment of depression is recommended by mental health professionals. Among the criteria for an MDD, at least five of the following symptoms must have been present during the 2-week period and at least one symptom must be "diminished interest/pleasure" or "depressed mood": (1) Depressed mood. For children and adolescents, this can also be an irritable mood; (2) Diminished interest or loss of pleasure in almost all activities; (3) Significant weight change or appetite disturbance. For children, this can be failure to achieve expected weight gain; (4) Sleep disturbance (insomnia or hypersomnia); (5) Psychomotor agitation or retardation; (6) Fatigue or loss of energy; (7) Feelings of worthlessness; (8) Diminished ability to think or concentrate, indecisiveness; (9) Recurrent thoughts of death, recurrent suicidal ideation without a specific plan, or a suicide attempt or specific plan for committing suicide [16,56. ICD-10 uses a list of 10 depressive symptoms and divides major depression into mild, moderate, or severe. In any of these cases, at least two of the three symptoms considered typical of depression must always be present; depressive mood, loss of interest and ability to enjoy and increase in fatigability and the duration of episodes should be 2 weeks $[15,55]$. ICD-10 seems more sensitive for the mild range of the depressive continuum than DSM-IV, while DSM-IV seems to be more sensitive for the moderate and severe ranges [57].

\section{Interaction effects between people with diabetes and depression}

\section{Glycemic control}

Depression can lead to impaired functioning and a loss of motivation, which can have a negative impact on self-management behavior. People with diabetes who mostly require long-term treatment are less likely to adhere to treatment, if depression co-exists. They can cause poor glucose control. In particular, depression and glycemic control in patients using insulin are closely related. Appropriate self-care is critical for good diabetes care. There is evidence that depression is associated with hyperglycemia in type 1 or type 2 diabetes [58-60]. A systematic review of treatment adherence among individuals with diabetes and depression indicated that there was a significant relationship between depression and treatment non-adherence [60]. Gonzalez et al. reported that patients with MDD adhere for significantly fewer days to diet, exercise and glucose self-monitoring self-management strategies. Logistic regression showed that major depression was associated with a 2.31 fold increase in the odds of missing one or more prescribed medications over the previous 7 days [59]. Diagnosis and treatment of depression lead to improved compliance to diabetes treatment and improved blood glucose control. In a Korean study, the odds ratio by CES-D scores was increased in the uncontrolled diabetic group with hemoglobin A1c higher than 10\% [61]. The consequences of poor blood glucose control may affect mental and psychological changes. Appropriate treatment of both diabetes and depression will lead to an improvement in both diseases.

\section{Diabetic complications and mortality}

Hyperglycemia has been linked to development of diabetic related complication. In a meta-analysis of 27 studies including people with type 1 and type 2 diabetes, depression was significantly associated with diabetic related complications including retinopathy, nephropathy, neuropathy, and sexual dysfunction [62]. Depression is associated with diabetic microvascular and macrovascular complications. However, it is unclear whether the increase in complications is a direct effect of depression or a consequence of comorbid depression. In the prospective cohort study, after adjustment for prior complication and demographic, clinical, and diabetes self-care variables, major depression was associated with higher risks of adverse microvascular outcomes (hazard ratio [HR], 1.36; 95\% confidence interval [CI], 1.05-1.75) adverse macrovascular outcomes (HR, 1.24; CI, 1.0-1.54) in the 5-year follow-up [63]. Recent studies have shown that coexisting de- 
pression increases mortality among people with diabetes. From the National Health and Nutrition Examination Survey (NHANES)-I Epidemiologic Follow-up Study, persons with diabetes with CES-D Scale scores of 16 or more showed 54\% greater mortality than those with scores under 16 , after controlling for sociodemographic, lifestyle, and health-status variables $(p=0.004)$ [64]. In the NHANES-I 8-year follow-up study, after adjusting for clinically relevant confounders, people with both diabetes and depression had a 1.3-fold increased risk of death from all causes compared with people with only diabetes, a 2-fold increased risk of death from all causes compared with people with only depression, and a 2.5 -fold increased risk of death compared with people without either diabetes or depression [65].

\section{CONCLUSION}

Improved understanding of diabetes and depression may potentially lead to early detection and timely treatment planning may prevent the onset of both disorders simultaneously. As reviewed recently, plausible mechanisms are postulated that involve shared biological pathways contributing to the pathogenesis of diabetes and depression (Fig. 1). Diabetes and depression may occur on the basis of common pathophysiology and interact with each other.

Currently, diabetic management cannot be successful unless the lifestyle and emotional status of the individual is considered. Unfortunately, the majority of people with this condition do not receive adequate care. People with diabetes who experience depressive symptoms should be screened for depression and be timely referred to mental health professionals. When we encounter patients with diabetes, they should be considered as people with diabetes and not patients with diabetes. It is necessary to understand the similarities and differences between depression of type 1 and type 2 diabetes. More research is required to investigate the direct links between diabetes and depression and the factors predicting and modifying depression in people with diabetes. Additionally, a paradigm shift in the management of diabetes with depression could greatly improve the quality of care and medical outcomes.
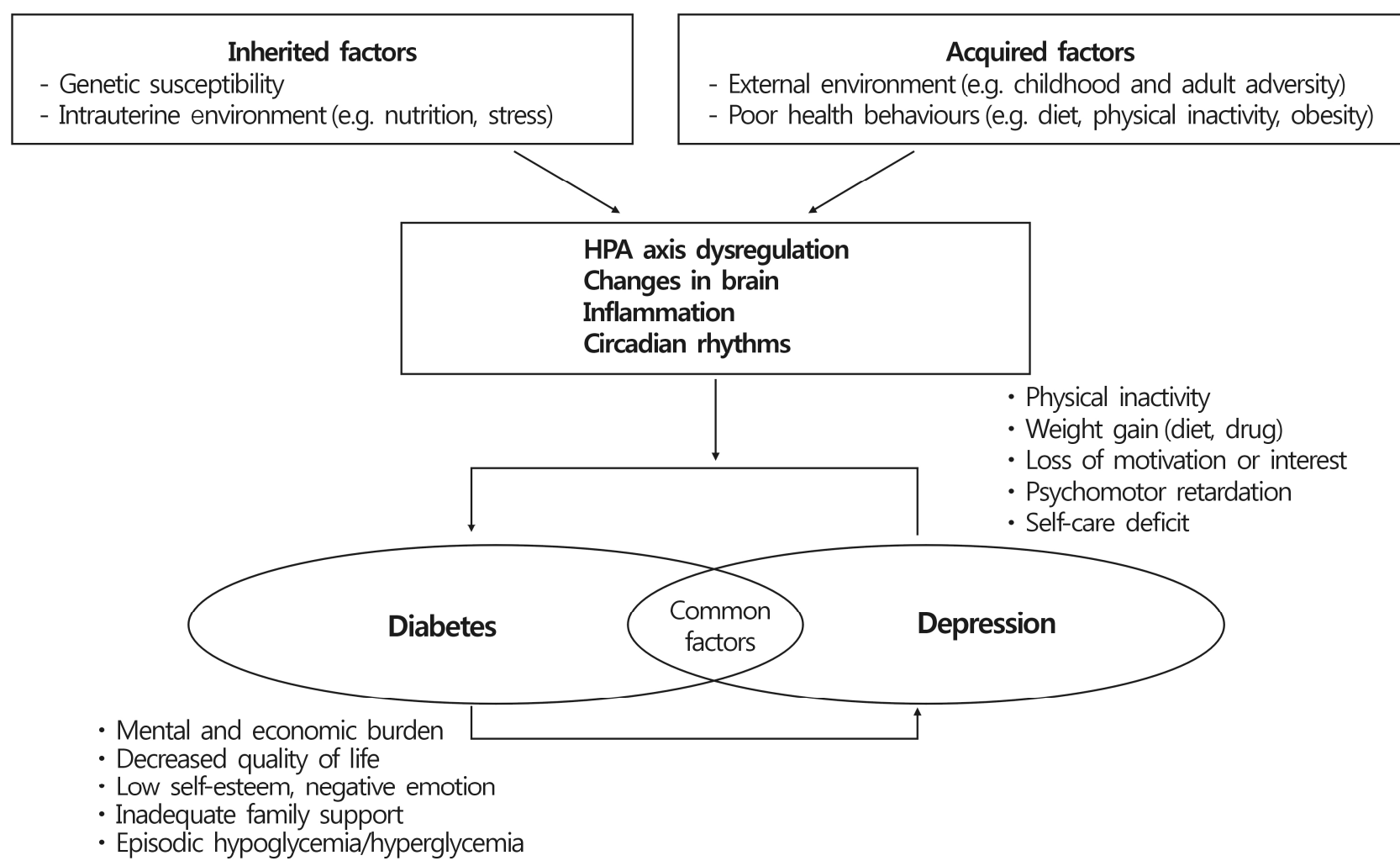

\section{Acquired factors}

- External environment (e.g. childhood and adult adversity)

- Poor health behaviours (e.g. diet, physical inactivity, obesity)

Fig. 1. Hypothesis of biological mechanisms of diabetes and depression. HPA, hypothalamus-pituitary-adrenal. 


\section{CONFLICT OF INTEREST}

No potential conflict of interest relevant to this article was reported.

\section{ORCHID}

Eon Ju Jeon, https://orcid.org/0000-0002-8858-5343

\section{REFERENCES}

1. International Diabetes Federation. IDF diabetes atlas. 8th ed. Brussels: International Diabetes Federation; 2017.

2. World Health Organization. Depression and other common. mental disorders: global health estimates. Geneva: World Health Organization; 2017.

3. Korean Diabetes Association. Diabetes fact sheet in Korea 2016 [Internet]. Seoul: Korean Diabetes Association; 2017 [cited 2018 April 18]. http://www.diabetes.or.kr/temp/KDA_fact_sheet $\%$ 202016.pdf

4. Tabák AG, Akbaraly TN, Batty GD, Kivimäki M. Depression and type 2 diabetes: a causal association? Lancet Diabetes Endocrinol 2014;2:236-45.

5 . Katon WJ. The comorbidity of diabetes mellitus and depression. Am J Med 2008;121(11 Suppl 2):S8-15.

6. IDF Clinical Guidelines Task Force. Global Guideline for Type 2 Diabetes: recommendations for standard, comprehensive, and minimal care. Diabet Med 2006;23:579-93.

7. American Psychiatric Association. Practice guideline for the treatment of patients with major depressive disorder. 3rd ed. Arlington: American Psychiatric Association; 2010.

8. Canadian Diabetes Association Clinical Practice Guidelines Expert Committee; Ekoé JM, Punthakee Z, Ransom T, Prebtani AP, Goldenberg R. Screening for type 1 and type 2 diabetes. Can J Diabetes 2013;37(Suppl 1):S12-5.

9. Young-Hyman D, de Groot M, Hill-Briggs F, Gonzalez JS, Hood K, Peyrot M. Psychosocial care for people with diabetes: a position statement of the American Diabetes Association. Diabetes Care 2016;39:2126-40.

10. Kahn CR, Weir GC, King GL, Moses AC, Smith RJ, Jacobson AM. Joslin's diabetes mellitus. 14th ed. Philadelphia: Lippincott Williams \& Willkins; 2005.

11. Dejgaard A, Gade A, Larsson H, Balle V, Parving A, Parving HH. Evidence for diabetic encephalopathy. Diabet Med 1991; 8:162-7.

12. Jesulola E, Micalos P, Baguley IJ. Understanding the pathophysiology of depression: from monoamines to the neurogenesis hypothesis model - are we there yet? Behav Brain Res 2018;341:79-90.

13. Nagy G, Ronai Z, Somogyi A, Sasvari-Szekely M, Rahman OA, Mate A, et al. P2RX7 Gln460Arg polymorphism is associated with depression among diabetic patients. Prog Neuro- psychopharmacol Biol Psychiatry 2008;32:1884-8.

14. Moulton CD, Pickup JC, Ismail K. The link between depression and diabetes: the search for shared mechanisms. Lancet Diabetes Endocrinol 2015;3:461-71.

15. World Health Organization. Pharmacological treatment of mental disorders in primary health care. Geneva: World Health Organization; 2009.

16. American Psychiatric Association. Diagnostic and statistical manual of mental disorders: DSM-5. 5th ed. Washington: American Psychiatric Association; 2013.

17. Willis T. Diabetes; a medical odyssey. Tuckahoe: USV Pharmaceutical Corp.; 1971.

18. Renn BN, Feliciano L, Segal DL. The bidirectional relationship of depression and diabetes: a systematic review. Clin Psychol Rev 2011;31:1239-46.

19. Grey M, Cameron ME, Lipman TH, Thurber FW. Psychosocial status of children with diabetes in the first 2 years after diagnosis. Diabetes Care 1995;18:1330-6.

20. Winkley K, Ismail K, Landau S, Eisler I. Psychological interventions to improve glycaemic control in patients with type 1 diabetes: systematic review and meta-analysis of randomised controlled trials. BMJ 2006;333:65.

21. Bennett DS. Depression among children with chronic medical problems: a meta-analysis. J Pediatr Psychol 1994;19:149-69.

22. Moulton CD, Costafreda SG, Horton P, Ismail K, Fu CH. Meta-analyses of structural regional cerebral effects in type 1 and type 2 diabetes. Brain Imaging Behav 2015;9:651-62.

23. Korczak DJ, Pereira S, Koulajian K, Matejcek A, Giacca A. Type 1 diabetes mellitus and major depressive disorder: evidence for a biological link. Diabetologia 2011;54:2483-93.

24. Lyoo IK, Yoon SJ, Musen G, Simonson DC, Weinger K, Bolo $\mathrm{N}$, et al. Altered prefrontal glutamate-glutamine-gamma-aminobutyric acid levels and relation to low cognitive performance and depressive symptoms in type 1 diabetes mellitus. Arch Gen Psychiatry 2009;66:878-87.

25. Lyoo IK, Yoon S, Jacobson AM, Hwang J, Musen G, Kim JE, et al. Prefrontal cortical deficits in type 1 diabetes mellitus: brain correlates of comorbid depression. Arch Gen Psychiatry 2012;69:1267-76.

26. Bolo NR, Musen G, Simonson DC, Nickerson LD, Flores VL, Siracusa T, et al. Functional connectivity of insula, basal ganglia, and prefrontal executive control networks during hypoglycemia in type 1 diabetes. J Neurosci 2015;35:11012-23.

27. Lustman PJ, Griffith LS, Clouse RE. Depression in adults with diabetes. Results of 5-yr follow-up study. Diabetes Care 1988; 11:605-12.

28. Eaton WW, Armenian H, Gallo J, Pratt L, Ford DE. Depression and risk for onset of type II diabetes. A prospective population-based study. Diabetes Care 1996;19:1097-102.

29. Lee HS, Lee JS, Lee HP, Jeon CE. Diabetes, Depression and doctor-patient relationship. Korean Diabetes J 2009;33:17882. Korean.

30. Krishnan V, Nestler EJ. The molecular neurobiology of depression. Nature 2008;455:894-902.

31. Knol MJ, Twisk JW, Beekman AT, Heine RJ, Snoek FJ, Pouwer F. Depression as a risk factor for the onset of type 2 diabetes 
mellitus:a meta-analysis. Diabetologia 2006;49:837-45.

32. Maes M. Cytokines in major depression. Biol Psychiatry 1994; 36:498-9.

33. Pickup JC. Inflammation and activated innate immunity in the pathogenesis of type 2 diabetes. Diabetes Care 2004;27: 813-23.

34. Germain A, Kupfer DJ. Circadian rhythm disturbances in depression. Hum Psychopharmacol 2008;23:571-85.

35. Reutrakul S, Van Cauter E. Interactions between sleep, circadian function, and glucose metabolism: implications for risk and severity of diabetes. Ann N Y Acad Sci 2014;1311:151-73.

36. Kan C, Pedersen NL, Christensen K, Bornstein SR, Licinio J, MacCabe JH, et al. Genetic overlap between type 2 diabetes and depression in Swedish and Danish twin registries. Mol Psychiatry 2016;21:903-9.

37. Anderson RJ, Freedland KE, Clouse RE, Lustman PJ. The prevalence of comorbid depression in adults with diabetes: a meta-analysis. Diabetes Care 2001;24:1069-78.

38. Beck AT, Steer RA, Brown GK. BDI-II, beck depression inventory: manual. 2nd ed. San Antonio: Psychological Corp.; Boston: Harcourt Brace; 1996.

39. Zauszniewski JA, Chung C, Krafcik K, Sousa VD. Psychometric testing of the depressive cognition scale in women with type 2 diabetes. J Nurs Meas 2001;9:61-72.

40. Colón de Martí LN, Guzmán Yunqué FS, Guevara-Ramos LM. Early detection of depression using the Zung Self-Rating Depression Scale. P R Health Sci J 1997;16:375-9.

41. Fisher L, Chesla CA, Mullan JT, Skaff MM, Kanter RA. Contributors to depression in Latino and European-American patients with type 2 diabetes. Diabetes Care 2001;24:1751-7.

42. Marathe PH, Gao HX, Close KL. American Diabetes Association Standards of Medical Care in Diabetes 2017. J Diabetes 2017;9:320-4.

43. Jones $\mathrm{A}$, Vallis $\mathrm{M}$, Pouwer F. If it does not significantly change $\mathrm{HbA1c}$ levels why should we waste time on it? A plea for the prioritization of psychological well-being in people with diabetes. Diabet Med 2015;32:155-63.

44. Deborah YH, Mark P. Psychosocial care for people with diabetes. Alexandria: American Diabetes Association; 2012. p. 240.

45. Lim SY, Lee EJ, Jeong SW, Kim HC, Jeong CH, Jeon TY, et al. The validation study of Beck Depression Scale 2 in Korean version. Anxiety Mood 2011;7:48-53.

46. Spitzer RL, Williams JB, Kroenke K, Linzer M, deGruy FV 3rd, Hahn SR, et al. Utility of a new procedure for diagnosing mental disorders in primary care. The PRIME-MD 1000 study. JAMA 1994;272:1749-56.

47. Kroenke K, Spitzer RL. The PHQ-9: a new depression diagnostic and severity measure. Psychiatr Ann 2002;32:509-15.

48. Shin JH, Kim HC, Jung CH, Kim JB, Jung SW, Cho HJ, et al. The Standardization of the Korean Version of the Patient Health Questionnaire-2. J Korean Neuropsychiatr Assoc 2013; 52:115-21. Korean.

49. Beck AT, Ward CH, Mendelson M, Mock J, Erbaugh J. An inventory for measuring depression. Arch Gen Psychiatry 1961;4:561-71.

50. Yoon SY, Lim JH, Han C. Rating scales for measurement- based clinical practice of depression. Korean J Psychopharmacol 2012;23:136-46. Korean.

51. Yesavage JA, Brink TL, Rose TL, Lum O, Huang V, Adey $\mathrm{M}$, et al. Development and validation of a geriatric depression screening scale: a preliminary report. J Psychiatr Res 19821983; 17:37-49.

52. Sheikh JI, Yesavage JA. Geriatric Depression Scale (GDS): recent evidence and development of a shorter version. In: Brink TL, editor. Clinical gerontology: a guide to assessment and intervention. New York: The Haworth Press; 1986. p. 165-73.

53. Bae JN, Cho MJ. Development of the Korean version of the Geriatric Depression Scale and its short form among elderly psychiatric patients. J Psychosom Res 2004;57:297-305.

54. Montorio I, Izal M. The Geriatric Depression Scale: a review of its development and utility. Int Psychogeriatr 1996;8:10312.

55. World Health Organization. ICD-10 Version: 2016 [Internet]. Geneva: World Health Organization; 2017 [cited 2018 April 18]. http://apps.who.int/classifications/icd10/browse/2016/en

56. Kwon JS, Kee NK, Bahk WM, Shin MS, Yu BH, YoonJS, et al. Diagnostic and statistical manual of mental disorders DSM. 5th ed. Seoul: Hakjisa; 2015.

57. Saito M, Iwata N, Kawakami N, Matsuyama Y, Ono Y, Nakane Y, et al. Evaluation of the DSM-IV and ICD-10 criteria for depressive disorders in a community population in Japan using item response theory. Int J Methods Psychiatr Res 2010; 19:211-22.

58. Lustman PJ, Anderson RJ, Freedland KE, de Groot M, Carney RM, Clouse RE. Depression and poor glycemic control: a meta-analytic review of the literature. Diabetes Care 2000; 23:934-42.

59. Gonzalez JS, Safren SA, Cagliero E, Wexler DJ, Delahanty L, Wittenberg E, et al. Depression, self-care, and medication adherence in type 2 diabetes: relationships across the full range of symptom severity. Diabetes Care 2007;30:2222-7.

60. Gonzalez JS, Peyrot M, McCarl LA, Collins EM, Serpa L, Mimiaga MJ, et al. Depression and diabetes treatment nonadherence: a meta-analysis. Diabetes Care 2008;31:2398-403.

61. Park HS, Hong YS, Lee HJ, Ha EH, Sung YA. The association between depressive symptoms and glycemic control in the patients with diabetes mellitus. Korean J Med 2003;64:204-10. Korean.

62. de Groot M, Anderson R, Freedland KE, Clouse RE, Lustman PJ. Association of depression and diabetes complications: a meta-analysis. Psychosom Med 2001;63:619-30.

63. Lin EH, Rutter CM, Katon W, Heckbert SR, Ciechanowski $\mathrm{P}$, Oliver MM, et al. Depression and advanced complications of diabetes: a prospective cohort study. Diabetes Care 2010; 33:264-9.

64. Egede LE, Nietert PJ, Zheng D. Depression and all-cause and coronary heart disease mortality among adults with and without diabetes. Diabetes Care 2005;28:1339-45.

65. Zhang X, Norris SL, Gregg EW, Cheng YJ, Beckles G, Kahn HS. Depressive symptoms and mortality among persons with and without diabetes. Am J Epidemiol 2005;161:652-60.

66. Kovacs M. Children's depression inventory (CDI2): technical manual. North Tonawanda: Multi-Health Systems; 2011. 